\title{
Performance Analysis of Battery Power Management Schemes in Wireless Mobile
}

\section{Devices}

\author{
Balakrishna J. Prabhu, A. Chockalingam and Vinod Sharma \\ Dept. of ECE, Indian Institute of Science, Bangalore, INDIA
}

\begin{abstract}
In this paper, we analyze the performance of battery power management schemes in wireless mobile devices using a queueing theory approach. We model the battery as a server with finite service capacity and data packets as customers to be served. With an intent to exploit the recharging capability of the battery when left idle, we allow the battery to go on intentional vacations during which the battery can recharge itself. The recharge thus built up can effectively increase the number of customers served (in other words, battery life can be extended). Such improved battery life performance would, however, come at the expense of increased packet delay performance. We quantify the battery life gain versus delay performance trade-off in this approach through analysis and simulations. By considering a continuous recharge model of the battery, we derive expressions for the number of customers served and the mean delay for an $M / G I / 1$ queueing system without and with server vacations. We show that allowing intentional vacations during busy periods helps to increase battery life, and that this approach can be beneficial when applied on traffic of delay-tolerant applications. We also propose a packet delay constrained power saving algorithm that will exploit the recharge phenomenon when packet delay constraints are imposed.
\end{abstract}

\section{INTRODUCTION}

Portable user terminals for mobile communications must rely on limited battery power for their operation [1]. It has been shown that energy savings in portable devices can be sought at different layers of the wireless protocol stack [2],[3], not necessarily at the devices/circuits level alone. Also, several studies characterizing the battery discharge behavior have shown that pulsed discharge performs better than continuous discharge [4]-[5]. Particularly, the battery can recharge itself (i.e., recover the potential) if left idle after discharge. This recharge phenomenon is illustrated in Fig. 1. Figure 1 shows the battery potential (in volts) as a function of time with continuous discharge and pulsed discharge. The parameter $d$ represents the duty cycle of the pulsed discharge pattern (i.e., $d=0.1$ means $10 \%$ discharge time followed by $90 \%$ relaxation time, and so on). The plots in Fig. 1 are generated using the battery simulation program developed by the Chemical Engineering department at UC, Berkeley [6]. A $2.76 \mathrm{~V}$ Lithium ion battery with a cutoff voltage of $1.9 \mathrm{~V}$ is considered. It is assumed that the battery ceases to deliver power once the voltage drops below the cutoff voltage. In other words, the time

This work was supported in part by the Department of Science and Technology, Govt. of India, New Delhi, under scheme III.5(32)/99-ET. taken for the battery to fall below the cutoff voltage is the battery life. The slope of battery discharge is determined by the discharge current density. Larger the value of this current density steeper will be the discharge slope, and hence lesser will be the time taken to reach the cutoff voltage. In Fig. 1 the discharge current density is taken to be $5 \mathrm{~A} / \mathrm{m}^{2}$.

From Fig. 1 it can be seen that if the battery is discharged continuously it takes about 7.5 minutes to reach the cutoff voltage. On the other hand, if the battery is discharged in pulsed mode, the battery recovers the voltage during the relaxation periods and it takes more time to reach the cutoff voltage. For example, if the duty cycle of the pulsed discharge is $50 \%$ (i.e., $d=0.5$ ) it takes more than 40 minutes to reach the cutoff voltage. Also, since the duty cycle is 0.5 , the total on-time of the battery is more than 20 minutes which is about three times the on-time in continuous discharge mode. As the discharge current density is taken to be the same in both continuous and pulsed discharge modes, this essentially means that the battery can deliver energy for a longer duration. This recharge effect advantage in pulsed mode can be exploited for improved energy efficiency in packet communications in wireless mobile devices.

The contribution in this paper is the proposal of methods to exploit the recharge phenomenon in batteries and the analysis of battery life gain and packet delay performance using a queueing theory approach. Recently, in [7] and [8] Chiasserini and Rao proposed a probabilistic recharge model for the battery. Through simulations, they showed that the recharge phenomenon can be exploited for battery life gain through suitable traffic shaping algorithms and battery level sensed routing strategies [8],[9].

In this paper, we model the battery as a server with finite capacity and the arriving packets at the mobile terminal as the customers to be served. Each transmitted packet consumes energy proportional to the packet size, transmission bit rate, wireless link design, etc. We propose to intentionally allow the server (battery) to go on vacation for calibrated amount of time, essentially allowing idle times for the battery to recharge itself. By doing so, the number of customers served can be increased (in other words, battery life can be increased). We derive expressions for the number of customers served and the average delay for an $M / G I / 1$ queueing system without and with server vacations. We show that allowing forced vacations 
during busy periods helps to increase battery life. However, since the customers (packets) have to wait in the queue when the server goes on vacation, the battery life gain will come at the expense of increased delay performance of the packets. It would be of interest to guarantee specified packet delay performances in practical systems. Hence, in order to achieve bounded delay performance, we further propose an algorithm that will exploit recharge phenomenon when packet delay constraints are imposed.

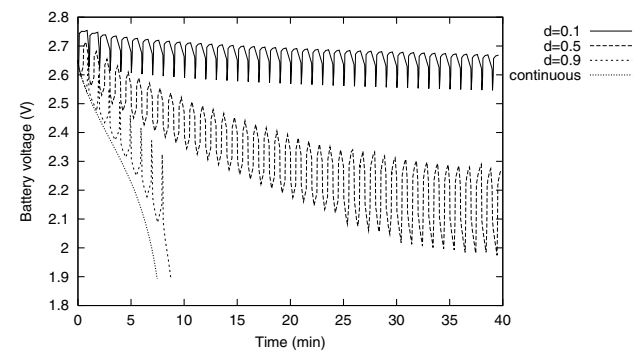

Fig. 1. Battery potential versus time for different duty cycles of discharge. Current density $=5 \mathrm{~A} / \mathrm{m}^{2}$. Cutoff voltage $=1.9 \mathrm{~V}$

\section{SySTEM MODEL}

Consider data packet transmission from a mobile terminal that draws power from a battery of finite capacity. We model the system as a queueing system with the battery as the server and packets as customers, as shown in Fig. 2. The packet interarrival time is assumed to be exponential (i.e., Poisson arrivals with rate $\lambda$ ) and service times are assumed to be i.i.d. with a general distribution. We assume that the battery has a nominal capacity of $N$ charge units. That is, if the transmission of a unit length packet consumes one charge unit and if the battery is discharged continuously, then the battery can serve $N$ such packets till it gets completely discharged.

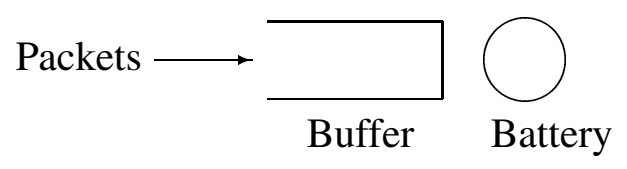

Fig. 2. Battery modeled as a server

\section{Battery Discharge/Recharge Model:}

In [7],[8], the battery discharge behavior is assumed to be discrete, i.e., each packet occupies a fixed slot size, and each packet transmission consumes one charge unit (or integer number of charge units). Also, in [7],[8] the battery recharge behavior (when the battery remains idle) too is modeled as a discrete process, i.e., the battery recharges by one charge unit with some probability if left idle for one slot duration. The recharge probability has been assumed to follow an exponential function that decreases with increasing discharged capacity. In other words, the recharge capability of the battery at any time is made proportional to the charge available in the battery at that time (i.e., more the available charge, more is the ability to recharge). In addition to the nominal capacity $N$, the battery is assumed to be characterized by another parameter called the theoretical capacity, $T$ charge units [7]. The battery is considered to be fully drained if either the available charge goes to zero or $T$ charge units of capacity has been delivered, which ever occurs first. Typically, the theoretical capacity of the battery is taken to be one or two orders higher than the nominal capacity [7].

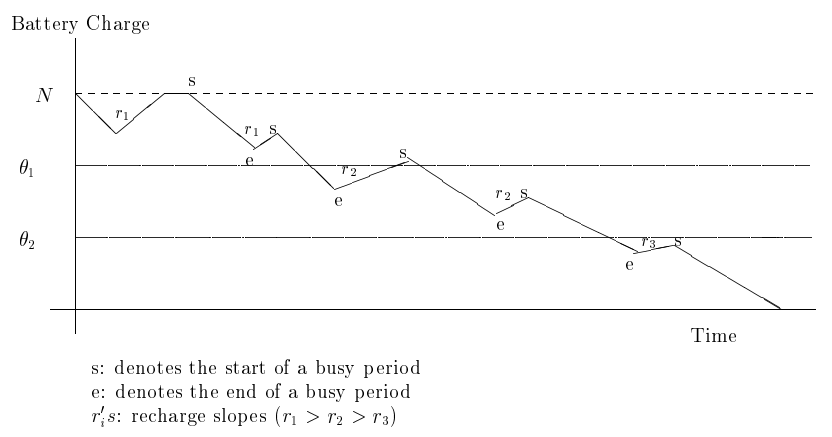

Fig. 3. Continuous discharge/recharge model

In this paper, we consider both the discharge as well as the recharge behavior of the battery as continuous phenomena, as illustrated in Fig. 3. While serving packets in busy periods, the battery looses charge linearly at a constant slope of unity. During idle periods, the battery recharges linearly with varying slopes depending on the battery level ${ }^{1}$ at the beginning of the idle period. The recharge model is more clearly explained as follows. We divide the range of charge from 0 to $N$ using $P$ threshold values, $\theta_{1}, \theta_{2}, \ldots, \theta_{P}$. The recharge slope is taken to be $r_{1}, r_{2}, \ldots, r_{P+1}$, respectively, when the battery level at the beginning of the idle period is in the range $\theta_{1}$ to $N, \theta_{2}$ to $\theta_{1}, \ldots, 0$ to $\theta_{P}$. By choosing $r_{1}>r_{2}>\ldots>r_{P+1}$, the model ensures that the ability to recharge reduces with decreasing battery level which is more realistic [6]. Thus, the parameters $P, \theta_{i}$ 's, and $r_{i}$ 's characterize the recharge behavior of the battery, which can be used in the mathematical analysis of the battery life gain due to recharge during idle/vacation periods.

\section{ANALYSIS}

We are interested in analyzing the battery performance in terms of mean number of packets served and mean packet delay when the battery's recharge behavior is exploited by intentionally allowing the battery to go on vacations.

\footnotetext{
${ }^{1}$ The term battery level is used to denote the amount of charge present in the battery.
} 
We first analyze and quantify the battery life gain due to idle periods (i.e., when buffer is empty) inherent in an $M / G I / 1$ queue. We then analyze a system where the server exhaustively serves all packets in the queue and takes intentional vacations before the start of the next busy period. We call this as a system with exhaustive service with vacations. We also propose a non-exhaustive service system with vacations where the server takes vacations during the busy period.

First consider a simple $M / G I / 1$ queue. Define a cycle as the time from start of one idle period to the start of the next idle period. A cycle consists of one idle and one busy period as shown in Fig. 4. Note that the first cycle starts after the first busy period. In order to obtain the expected number of customers served, we

1) obtain the distribution of the amount of charge left at the end of the first busy period,

2) obtain the expected number of cycles after the first busy period till the charge goes to zero, and

3) obtain the expected number of customers served in each cycle,

as follows.

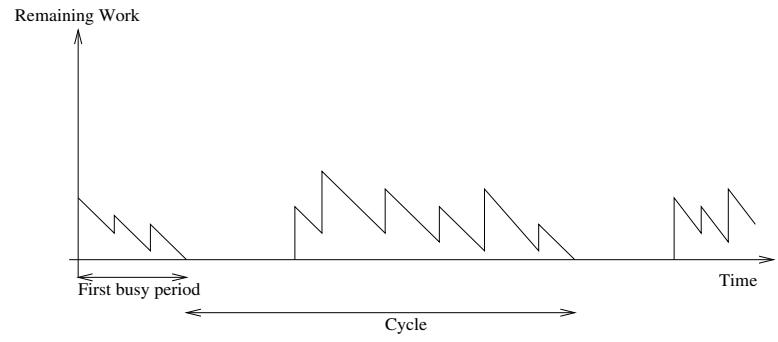

Fig. 4. Definition of a cycle in the system

For the $M / G I / 1$ queue the idle period is distributed as $\exp (\lambda)$. Let

$$
Z_{i}=Y_{i}-B_{i}
$$

where $B_{i}$ is the charge consumed during the busy period and $Y_{i}$ is the amount of recharge during the idle period in the $i^{t h}$ cycle, respectively. Then, $Z_{i}$ is the total charge lost $\left(Y_{i}<B_{i}\right)$ or gained $\left(Y_{i}>B_{i}\right)$ in the $i^{\text {th }}$ cycle. Let $\tau$ be the random variable denoting the number of cycles till the charge goes to zero, and let $z$ be a random variable which denotes the charge at the end of the first busy period. We need to find

$$
\tau=\inf \left\{n: \sum_{i=1}^{n} Z_{i} \leq-z\right\}
$$

Let $E_{z}[\tau]$ denote the expected number of cycles given that charge after the first busy period is $z$. We can then write the integral equation

$$
E_{z}[\tau]=P\left[Z_{1} \leq-z\right]+\int_{-z}^{N-z}\left(1+E_{z_{1}}[\tau]\right) \cdot d F_{Z_{1}}\left(z_{1}\right)
$$

$$
\begin{aligned}
& =P\left[Z_{1} \leq N-z\right]+\int_{-z}^{N-z} E_{z_{1}}[\tau] \cdot d F_{Z_{1}}\left(z_{1}\right) \\
& =1+\int_{-z}^{N-z} E_{z_{1}}[\tau] \cdot d F_{Z_{1}}\left(z_{1}\right),
\end{aligned}
$$

where $F_{Z_{1}}\left(z_{1}\right)$ is the cdf of $Z_{1}$. To obtain the distribution of $Z_{i}$, we need to obtain the distributions of $Y_{i}$ and $B_{i}$.

The distribution of $Y_{i}$ is obtained as follows. Let $T_{i}$ denote the duration of the idle period of the $i^{t h}$ cycle and $z^{\prime}$ denote the charge at the beginning of the $i^{t h}$ cycle. Then

$$
Y_{i}=\min \left(r_{k} T_{i}, N-z^{\prime}\right)
$$

and

$$
F_{Y_{i}}(y)= \begin{cases}F_{T_{i}}\left(y / r_{k}\right) & y \leq N-z^{\prime} \\ 1 & y>N-z^{\prime}\end{cases}
$$

where $k \in\{1,2, \ldots, P+1\}$ is determined by the charge threshold values between which $z^{\prime}$ lies. Since $T_{i}$ is distributed as $\exp (\lambda)$, the cdf of $Y_{i}$ is given by

$$
F_{Y_{i}}(y)= \begin{cases}1-e^{-\frac{\lambda y}{r_{k}}} & y \leq N-z^{\prime} \\ 1 & y>N-z^{\prime}\end{cases}
$$

Computing the distribution of $B_{i}$ for a general $M / G I / 1$ queue may not be so easy. Here, we limit ourselves to exponential service times with rate $\mu$. Then, $B_{i}$ has density [10]

$$
f_{B_{i}}(b)=\frac{I_{1}(2 b \sqrt{\lambda \mu}) \cdot e^{-b(\lambda+\mu)}}{b \sqrt{\rho}}
$$

where $I_{1}(\cdot)$ is the modified Bessel function of the first order, and $\rho=\frac{\lambda}{\mu}$. The cdf of $Z_{1}, F_{Z_{1}}\left(z_{1}\right)$ can then be written as

$$
\begin{aligned}
F_{Z_{1}}\left(z_{1}\right)= & \int_{\max \left(0,-z_{1}\right)}^{\infty} F_{Y_{1}}\left(z_{1}+b\right) f_{B_{1}}(b) d b \\
= & \int_{\max \left(0,-z_{1}\right)}^{N-z-z_{1}} F_{Y_{1}}\left(z_{1}+b\right) f_{B_{1}}(b) d b+\int_{N-z-z_{1}}^{\infty} f_{B_{1}}(b) d b .
\end{aligned}
$$

Eqn. (3) can be numerically solved to obtain $E_{z}[\tau]$. To obtain $E[\tau]$ we average $E_{z}[\tau]$ over $z$, as

$$
E[\tau]=\int_{0}^{N} E_{N-z}[\tau] d F_{B_{0}}(z)
$$

where $F_{B_{0}}$ is the distribution of the first busy period. The expected number of customers served can be obtained as the 
product of the expected number of cycles and the expected number of customers served in a busy period as follows ${ }^{2}$

$$
C=(1+E[\tau]) \frac{1}{1-\rho} .
$$

Note that the expected number of customers served is upper bounded by the theoretical capacity of the battery, $\mu T$. The mean delay of packets in the $M / M / 1$ queue is given by [11]

$$
\bar{W}=\frac{\lambda}{\mu^{2}(1-\rho)} .
$$

\section{A. Exhaustive Service System with Vacations}

Next, we consider a system where the server takes intentional vacations. The server exhaustively serves all packets in the queue and takes a vacation. If after completion of vacation, there is still no packet in the queue, it takes another vacation, and so on. We assume the vacation time distribution to be $\exp (\delta)$, where $\delta$ can be a system parameter.

We proceed in similar fashion as before to derive the expected number of customers served and mean delay. In addition to idle times in a simple $M / G I / 1$ queue, the vacation times too contribute to the idle time distribution in this case. The idle time distribution of this system can be shown to be $\exp (p \delta)$, where $p=\frac{\lambda}{\lambda+\delta}$. The distribution of $Y_{i}$ in this system with vacation is the same as that given in Eqn. (5). In the system without vacation, the busy period starts as soon as a customer arrives following an idle period. However, in the system with vacations considered in this subsection, the busy period may start after queuing up a random number of customers. Hence, to find the distribution of the busy period in a cycle we need to condition on the number of customers waiting before the start of the busy period. The Laplace transform of the busy period in a cycle, considering the special case of exponential service times, can be written as

$$
\mathcal{B}^{\prime}(s)=\sum_{n=1}^{\infty}[\mathcal{B}(s)]^{n} p(n),
$$

where $\mathcal{B}(s)$ is the Laplace transform of the busy period of a simple $M / M / 1$ queue, and $p(n)$ is the probability of $n$ customers waiting at the start of a busy period. The expression for $\mathcal{B}(s)$ can be written as [10]

$$
\mathcal{B}(s)=\frac{\mu+\lambda+s-\sqrt{(\mu+\lambda+s)^{2}-4 \lambda \mu}}{2 \lambda} .
$$

The expression for $p(n)$ can be derived as follows.

$$
P(\text { at least one arrival occurs during a vacation })=\frac{\lambda}{\lambda+\delta},
$$

$P(n$ arrivals occur in a vacation $)=\delta \int_{0}^{\infty} \frac{(\lambda v)^{n}}{n !} e^{(-(\lambda+\delta) v)} d v$.

\footnotetext{
${ }^{2}$ Actually this is an approximation (in fact, an upper bound) because the last cycle may not be complete
}

Hence,

$P(n$ arrivals occur given at least one arrival occurs $)=$

$$
\frac{\delta}{p} \int_{0}^{\infty} \frac{(\lambda v)^{n}}{n !} e^{(-(\lambda+\delta) v)} d v
$$

From Eqns. (12), (13), (14), $\mathcal{B}^{\prime}(s)$ can be obtained as,

$$
\mathcal{B}^{\prime}(s)=\frac{\delta \mathcal{B}(s)}{\lambda+\delta-\lambda \mathcal{B}(s)} .
$$

The pdf of the busy period, $f_{B^{\prime}}$, can then be obtained by inverting the above equation as [12]

$$
\begin{array}{r}
f_{B^{\prime}}(b)=\frac{4(\lambda+\delta) \sqrt{\lambda \mu} \delta}{\lambda} \cdot \frac{e^{-(\lambda+\mu) b}}{\sqrt{b}} \\
\int_{0}^{\infty} \frac{e^{-2(\lambda+\delta) u} I_{1}(2 \sqrt{\lambda \mu b(b+2 u)}) u}{\sqrt{b+2 u}} d u .
\end{array}
$$

From the above distributions of idle period, recharge, and busy period, the expected number of cycles $E[\tau]$ can be obtained from Eqns. (3) and (9). The expected number of customers served in the first busy period is $(1-\rho)^{-1}$. The expected number of customers served in the subsequent busy periods is given by $\left(\frac{\lambda+\delta}{\delta}\right)\left(\frac{1}{1-\rho}\right)$. The expected number of customers served can then be written as

$$
C=\frac{1}{1-\rho}+E[\tau]\left(\frac{\lambda+\delta}{\delta}\right)\left(\frac{1}{1-\rho}\right) .
$$

Here again, the expected number of customers served is upper bounded by the theoretical capacity of the battery. The mean delay for this system with vacations can be written as [11]

$$
\bar{W}=\frac{\lambda}{\mu^{2}(1-\rho)}+\frac{1}{\delta} .
$$

\section{B. Non-exhaustive system with vacations}

Another way to improve battery life is to interrupt service during busy period and allow vacations. Specifically, we allow the server to go on vacation after serving $K$ packets in a row during a busy period. We assume the duration of this vacation to be i.i.d and distributed as $\exp (\delta)$. Both $\delta$ and $K$ are parameters which can be chosen to allow desired vacation times.

In order to analyze this scheme, consider an approximate system which models the non-exhaustive system with vacations at high arrival rates. Assume the arrival rate is high so that there are always packets to send in the queue. In this case, we can take the busy period to be the sum of $K$ service times. Thus, for the case of $\exp (\mu)$ service times the pdf of the busy period can be written as

$$
f_{B_{i}}(b)=\frac{\mu^{K} b^{(K-1)} e^{-\mu b}}{(K-1) !} .
$$


The vacation period is distributed as $\exp (\delta)$. We then use Eqns. (5), (3), and (9) to obtain the expected number of cycles to discharge completely, $E[\tau]$. The number of packets served $C$ is then given by,

$$
C=\frac{K E[\tau]}{\mu} .
$$

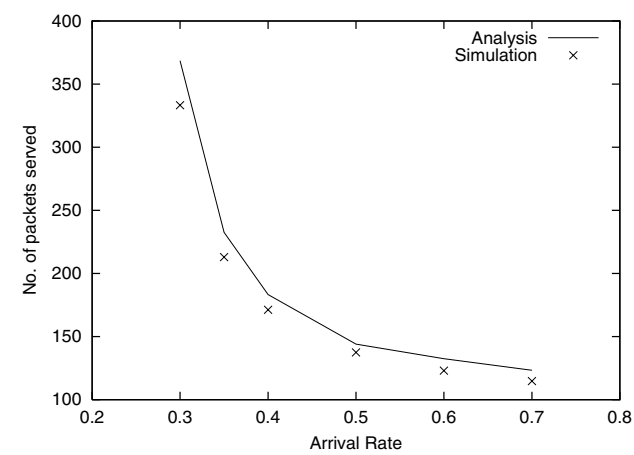

Fig. 5. Number of packets served versus arrival rate in the system withou vacations. $N=100 . \theta_{1}=75, \theta_{2}=50, \theta_{3}=25 . r_{1}=0.4, r_{2}=0.3$, $r_{3}=0.2, r_{4}=0.1 . \mu=1$.

\section{RESUlTS AND DiscuSSION}

We consider the following system parameters: nominal capacity $N=100$ charge units, number of thresholds $P=3$, threshold values $\theta_{1}=75, \theta_{2}=50, \theta_{3}=25$, recharge slopes $r_{1}=0.4, r_{2}=0.3, r_{3}=0.2, r_{4}=0.1$, and service time parameter $\mu=1$. First, from Eqn. (10), we compute the number of packets served in a simple $M / M / 1$ queue with battery recharge due to inherent idle periods. Figure 5 shows the number of packets served for different arrival rates $\lambda$, obtained through analysis and simulation. In the numerical evaluation of Eqn. (3), the integral is discretized in steps of 0.1 and converted into a set of linear equations. Further, instead of evaluating all the entries of the coefficient matrix we compute the entries for one row and use the same for the other rows for a given threshold region. This significantly reduces the computation time while maintaining the accuracy of the results as observed from the close match between the analytical and simulation results. It can be observed that the recharge due to inherent idle periods can increase the number of customers served to about three times the battery's nominal capacity of 100 charge units, particularly at low arrival rates (e.g., 350 packets served at $\lambda=0.3$ ).

Next, in Fig. 6, we plot the number of customers served in the system with intentional vacations and exhaustive service (as per Eqn. (17)) as described in Section III-A. The corresponding mean delay performance is plotted in Fig. 7. The performance curves are parameterized by the vacation time parameter $\delta$. The performance of the system without vacation (as per Eqn. 10) is also plotted for comparison. It is observed that allowing additional vacations during idle periods does not provide significant increase in the number of customers served. For example, when $\delta=1$ the number of customers served increases only by less than $1 \%$. When the vacation times are made larger (smaller values of $\delta, \delta=0.05,0.1$ ) the number of packets served, in fact, reduces when compared to the system without vacations. This behavior can be attributed to two reasons: a) even if large vacation times are given, the battery will not be able to recharge beyond its nominal capacity $N$, and $b$ ) increasing vacation times allows more packets to queue up resulting in longer busy periods which depletes the battery charge faster. The mean delay increases with increasing mean vacation time as seen in Fig. 7. The usefulness of the above observation is that leaving vacation times in an exhaustive service system is not effective and alternate schemes to exploit the recharge effect need to be looked into. One such alternate approach is to start service as soon as the battery recharges to its nominal capacity. The performance of this approach has been evaluated using simulations and the resulting number of packets served for different values of $\delta$ are plotted in Fig. 8. It is observed that this approach results in moderate gains in number of packets served at moderate arrival rates (in the range $\lambda=0.3$ to 0.5 ). However, at high arrival rates there is no significant gain. Consequently, we investigate yet another approach which is the non-exhaustive system with vacations as described in Section III-B.

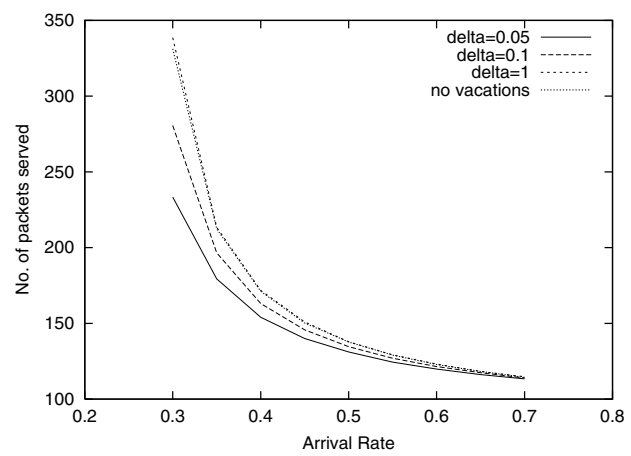

Fig. 6. Number of packets served versus arrival rate in the exhaustive service system with vacations for different values of $\delta . N=100 . \theta_{1}=75, \theta_{2}=50$, $\theta_{3}=25 . r_{1}=0.4, r_{2}=0.3, r_{3}=0.2, r_{4}=0.1 . \mu=1$.

Figures 9 and 10 illustrate the number of customers served and the mean delay performance of the non-exhaustive service system with vacations for various arrival rates obtained through simulations. The plots are parameterized with parameter $K$ for $\delta=0.05$. It is observed that for low arrival rates both the systems without and with vacations perform similar, reaching the theoretical capacity of the battery, $T$, which is taken to be $10 N$. For example, when the arrival rate is quite low $(\lambda=0.2)$ the inherent idle periods themselves will allow sufficient recharge to deliver the theoretical capacity, and therefore allowing additional vacation times has no 


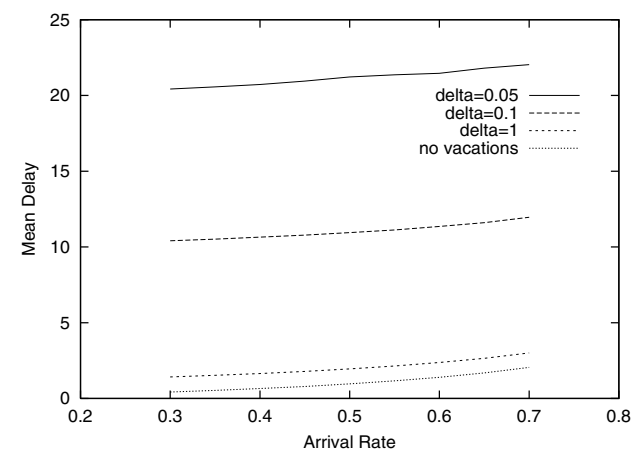

Fig. 7. Mean delay versus arrival rate in the exhaustive service system with vacations for different values of $\delta . N=100 . \theta_{1}=75, \theta_{2}=50, \theta_{3}=25$. $r_{1}=0.4, r_{2}=0.3, r_{3}=0.2, r_{4}=0.1 . \mu=1$.

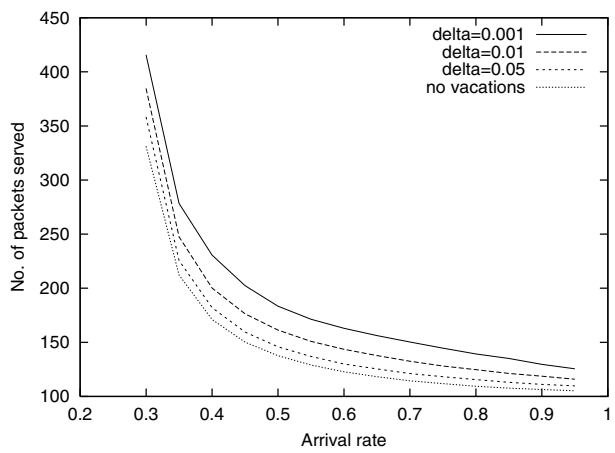

Fig. 8. Number of packets served versus arrival rate in the exhaustive service system with vacations limited to time to recharge to $N$ for different values of $\delta . N=100 . \theta_{1}=75, \theta_{2}=50, \theta_{3}=25 . r_{1}=0.4, r_{2}=0.3, r_{3}=0.2$, $r_{4}=0.1 . \mu=1$.

effect. However, at high arrival rates the system with vacations increasingly serves more packets than the system without vacations for increasing values of $K$. For example, taking a vacation each time a customer from the queue is served (i.e., $K=1$ ) the theoretical capacity can be reached even at high arrival rates. For larger values of $K$ the number of packets served initially decreases with increasing arrival rate up to some point after which the number of packets served increases. This can be explained as follows. At low arrival rates the number of packets in the queue will be less and hence the probability of taking vacations will be less leading to lesser recharge. However, for large arrival rates the buffer will be mostly occupied leading to more vacations and more recharge. The mean delay performance gets increasingly worse with lower values of $K$. Though $K=1$ and 2 result in close to theoretical capacity in terms of number of customers served, the delay for $K=2$ is significantly lower than that of $K=1$, indicating the possibility of choice of the parameters of $K$ and $\delta$ that can result increased battery life with less degradation in delay. Thus the non-exhaustive service system with vacations can be applied on the traffic of delay-tolerant applications (email, file transfers, etc.) to increase battery life in wireless mobile devices.

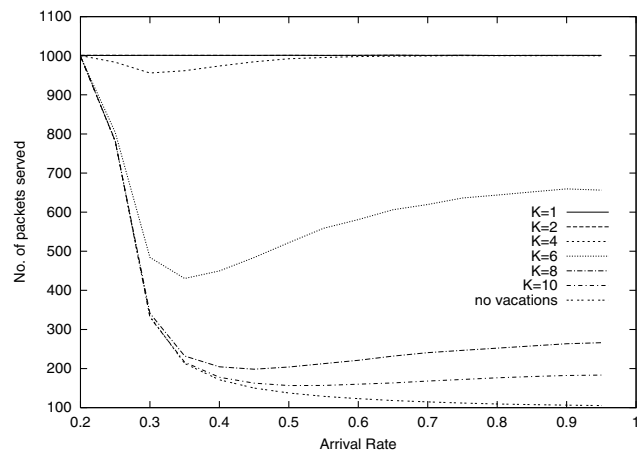

Fig. 9. Number of packets served versus arrival rate in the non-exhaustive service system with vacations for different values of $K . N=100 . \theta_{1}=75$, $\theta_{2}=50, \theta_{3}=25 . r_{1}=0.4, r_{2}=0.3, r_{3}=0.2, r_{4}=0.1 . \mu=1$. $\delta=0.05$

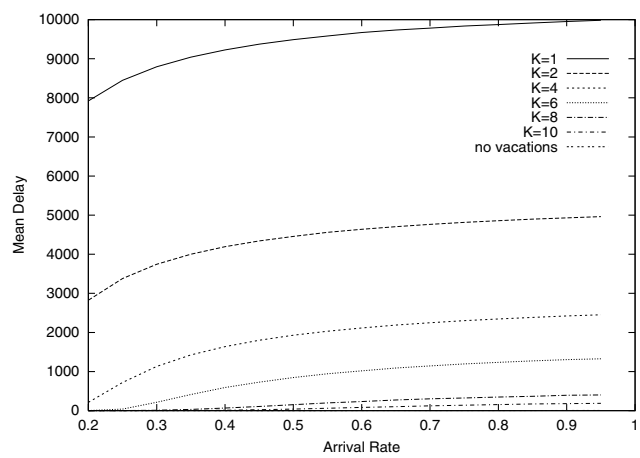

Fig. 10. Mean delay versus arrival rate in the non-exhaustive service system with vacations for different values of $K . N=100 . \theta_{1}=75, \theta_{2}=50$, $\theta_{3}=25 . r_{1}=0.4, r_{2}=0.3, r_{3}=0.2, r_{4}=0.1 . \mu=1 . \delta=0.05$.

\section{Delay Constrained Power Saving}

The proposed schemes in Section III resulted in increased battery life at the expense of increased packet delay performance. The packets delays are found to increase monotonically with increasing intentional relaxation times. It would be of interest to guarantee specified packet delay performances in practical systems. Hence, in order to achieve bounded delay performance we consider the following algorithm for serving packets.

- Consider Poisson arrival of packets with rate $\lambda$ and exponential service times with mean $\mu-1$, so that $\rho=\lambda / \mu$.

- The device maintains a count of the total number of packets, $N_{s}$, served so far and the sum of the delays experienced by each packet, $D_{s}$.

- After serving $K$ packets the device computes the number of packets, $N_{c}$, which when served continuously will discharge the battery to its cutoff voltage. We can approximate the battery potential to be a linearly decreasing function of time when discharged at a constant current density. This discharge slope can be obtained a priori 
from the battery discharge behavior as plotted in Fig. 1. Using the knowledge of the present battery voltage and the discharge slope, the device can calculate the time it will take to reach the cutoff voltage and hence the number of packets it can serve continuously, $N_{c}$, and the sum of the delays that will be experienced by these packets when served continuously, $D_{c}$.

- The device then calculates the average delay $\bar{D}$ as, $\bar{D}=$ $\frac{D_{s}+D_{c}}{N_{s}+N_{c}}$.

- Let $D$ be the delay constraint imposed in the system. If $\bar{D} \geq D$ then the battery stops taking further vacations and serves the remaining packets in a continuous fashion till the cutoff voltage is reached. On the other hand, if $\bar{D}<D$ then the battery continues to take a vacation for every $K$ packets served.

The performance of this delay constrained algorithm has been evaluated using the battery simulation program from Berkeley [6]. Figures 11 and 12 show the number of packets served and mean delay performance of the system with vacations and delay constraint. The system performance without vacations is also plotted for comparison. The packet arrival process is Poisson with rate $\lambda$ and the service time is exponential with mean $\mu^{-1}=0.02 \mathrm{sec}$. The vacation time is taken to be exponential with rate $\delta=0.05 \mu$. K is chosen to be equal to 4 . The current density is taken as $10 \mathrm{~A} / \mathrm{m}^{2}$. It is seen that the system with vacation results in increased number of packets served compared to the system without vacation. Also, as the delay constraint is relaxed (e.g., from $D=5$ secs to $D=10$ secs) the number of packets served is increased. For example, when $\rho=0.5$ the system without vacation serves about 135 packets whereas the system with vacations serves about 160 and 240 packets for $D=5$ secs and $D=10$ secs, respectively. Also, from Fig. 12 it can be observed that the proposed algorithm ensures that the mean delay performance never exceeds the imposed delay constraint.

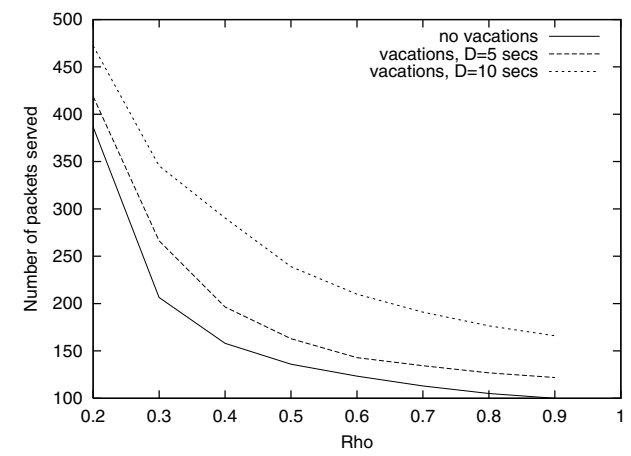

Fig. 11. Number of packets served vs. $\rho=\lambda / \mu . \mu=0.02$ sec. $\delta / \mu=$ 0.05. $K=4$.

\section{Conclusions}

We analyzed the performance of battery power management schemes in wireless mobile devices using a queueing theory

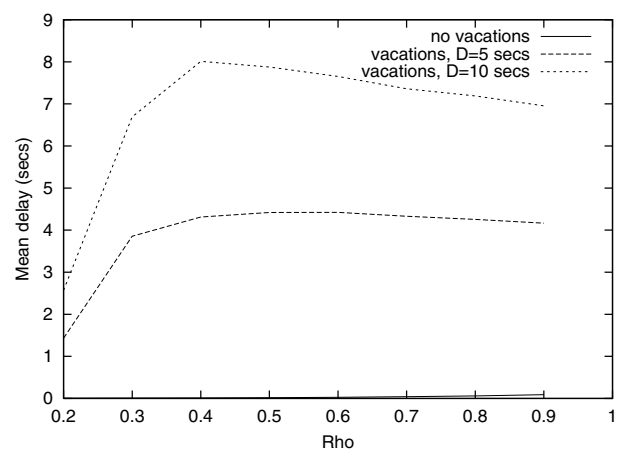

Fig. 12. Mean delay vs. $\rho=\lambda / \mu . \mu=0.02$ sec. $\delta / \mu=0.05 . K=4$.

approach. We modeled the battery as a server with finite service capacity and proposed schemes to allow intentional server vacations to exploit the battery recharge effect for increased battery life. We analyzed and quantified the increase in battery life due to idle and vacation periods in a $M / G I / 1$ queue. Systems with vacations having exhaustive and non-exhaustive service were considered. We showed that a non-exhaustive service system with vacations can result in significant gain in battery life, and that this approach can be beneficial when applied on traffic of delay-tolerant applications in wireless mobile devices. We also proposed an algorithm that exploited the recharge phenomenon when packet delay constraints are imposed.

\section{REFERENCES}

[1] "Energy management in personal communications and mobile computing," special issue, IEEE Personal Commun., June 1998.

[2] A. Chockalingam and M. Zorzi, "Energy efficiency of media access protocols for mobile data networks," IEEE Trans. on Commun., vol. 46, no.11, pp. 1418-1421, November 1998.

[3] W. Heinzelman, A. Chandrakasan, and H. Balakrishnan, " Energy efficient routing protocols for wireless microsensor networks," Proc. $33^{\text {rd }}$ Hawaii Intl. Conf. on System Sciences (HICSS'00), January 2000.

[4] R. M. Lafollette and D. Bennion, "Design fundamentals of high power density, pulsed discharge, lead-acid batteries. II: Modeling," Jl. Electrochem. Soc., vol. 137, no. 12, pp. 3701-3707, December 1990.

[5] B. Nelson, R. Rinehart, and S. Varley, "Ultrafast pulsed discharge and recharge capabilities of thin-metal film battery technology," $11^{\text {th }}$ IEEE Intl. Pulsed Power Conf., pp. 636-641, June 1997.

[6] J. S. Newman, FORTRAN programs for simulation of electrochemical systems. http://www.cchem.berkeley.edu/jsngrp/

[7] C. F. Chiasserini and R. R. Rao, "Pulsed battery discharge in communication devices," Proc. Mobicom'99, August 1999.

[8] C. F. Chiasserini and R. R. Rao, "A traffic control scheme to optimize the battery pulsed discharge," Proc. IEEE MILCOM'99, November 1999.

[9] C. F. Chiasserini and R. R. Rao, "Energy efficient battery management," Proc. INFOCOM 2000, March 2000.

[10] L. Kleinrock, Queueing Systems Vol. 1:Theory. John Wiley, 1975.

[11] D. Bertsekas and R. Gallager, Data Networks. Prentice-Hall, Englewood Cliffs, New Jersy, 1987.

[12] G. E. Roberts and H. Kaufman, Table of Laplace Transforms W. B. Saunders Company, 1966. 